\title{
Research Progress on Self-Care Ability of Hemodialysis Patients
}

\author{
Xiaojiao Zhang, Chenyun Xu \\ Hainan General Hospital, Haikou, China \\ Email: g2002m@163.com
}

How to cite this paper: Zhang, X.J. and $\mathrm{Xu}$, C.Y. (2021) Research Progress on Self-Care Ability of Hemodialysis Patients. Open Journal of Nursing, 11, 320-330. https://doi.org/10.4236/ojn.2021.115029

Received: April 15, 2021

Accepted: May 23, 2021

Published: May 26, 2021

Copyright $\odot 2021$ by author(s) and Scientific Research Publishing Inc. This work is licensed under the Creative Commons Attribution International License (CC BY 4.0).

http://creativecommons.org/licenses/by/4.0/

\begin{abstract}
Good self-management can help hemodialysis patients improve their quality of life and survival rate. This article reviews the status quo of self-care ability of maintenance hemodialysis patients, the influencing factors of self-care ability and the intervention measures. To improve the self-care ability and quality of life of patients, coordinated nursing intervention, self-efficacy intervention, psychological intervention and health education were proposed. To provide a reference for the self-management of hemodialysis patients.
\end{abstract}

\section{Keywords}

Hemodialysis, Self-Care, Influencing Factors, Intervention

\section{Introduction}

Maintenance hemodialysis (MHD) is one of the main methods of renal replacement therapy in patients with end stagerenal disease (ESRD). With the continuous improvement of dialysis technology, although the physiological function of MHD patients has been improved, their survival rate and quality of life are still poor due to the long-term nature of treatment and the particularity of the disease [1]. The longer the duration of dialysis treatment is, the greater the physiological and psychological pressure that hemodialysis patients face, which will to some extent affect the ability of patients to self-care. Self-care ability refers to the ability that people acquire through learning to meet their own needs and related care. This ability is a strategy for coping with life events and pressures and improving the quality of life [2]. Therefore, to MHD patients, improving the level of self-care ability has a positive effect on improving the survival rate and quality of life. This article reviews the status quo of self-care ability of MHD patients, the related factors affecting self-care ability and the intervention measures. 


\section{The Concept and Connotation of Self-Care and Self-Care Ability}

\subsection{Self-Care}

Self-care as a concept in Orem's theory of self-care is the ability of an individual to sustain life in a stable or changing environment, a series of voluntary regulatory actions and self-care activities to ensure structural integrity and functional well-being and to promote health and well-being [3]. Orem's view is that self-care related to personal health should be the responsibility of the individual, and care interventions are taken solely to help people improve their ability to care for themselves in order to improve their health and quality of life [4]. Self-care is related to many factors, such as people, living environment, social culture and life value, which can be used as a positive behavior guidance for people's practice and disease treatment [5]. In 1998, WHO defined the meaning and content of self-care, and put forward that self-care is a measure and action taken by individuals in order to obtain health, prevent disease and treat disease. It includes nutrition, hygiene, environment, lifestyle, social and economic factors, and selfmedication.

\subsection{Self-Care Ability}

In 1971, the American nurse Scientist Orem proposed the Self-Care Agency [6] [7]. It is one of the concepts in the self-care theory system. The concept of self-care ability has been studied and developed by many scholars in different areas, from Fleischer and Kearny's study in 1979 to Denyes's study in 1980 [8] [9], Hanson and Bickeltl in 1985, the concept of "the ability of an individual to engage in self-care" is widely accepted as a means of meeting the needs of individual health activities [10]. The implication is: after cognitive and psychological regulation, to take appropriate assessment intervention, so that the individual behavior reasonable implementation, to ensure the individual's health, maintain good emotional control, use of internal and external environmental resources, to stay healthy. Self-care is an act performed by individuals to protect their life, health, and well-being [11]. The person who can well meet the needs of self-care is the person who has the appropriate self-care ability and takes responsibility for self-health. The patient carries on the daily life when does not depend on other people, enhances the individual to the life satisfaction.

\section{With the Change of Bio-Psycho-Social Model, the Self-Care Ability of MHD Patients Is Becoming Stronger and Stronger}

In order to maintain and promote their own health, they can not rely entirely on the help of others, more of a need for self care and self care [12]. Self-care of MHD patients includes Diet and water intake, symptom management, maintenance of arteriovenous Fistula, medication and exercise instruction. Except for regular dialysis, ESRD patients need to take care of themselves at home most of 
the time, but the longer the dialysis treatment lasts, the worse the self-care ability of the patients, and some daily activities need to rely on the help of others. The results of investigation on $100 \mathrm{MHD}$ patients and their primary caregivers show that improving the self-care ability of the patients not only promotes the physical and mental health of the patients [12], but also has a positive effect on the physical and mental health of their primary caregivers. The results showed that the total score of self-care ability of MHD patients was (105.12 \pm 16.81$)$ [13], which was in the middle and low level, the self-care ability of $78.3 \%$ of patients was in the middle level, of which $33.9 \%$ patients had low self-care ability in Diet and daily life nursing Kgriva et al. [14]. Investigated the self-care ability of 195 hemodialysis patients and its influencing factors [15]. The results showed that the total score of self-care ability and the scores of each dimension of hemodialysis patients were in middle and high level, the reason may be related to the difference of the patient's area and living habit. Han [16] and other scholars investigated the self-care behavior of 113 elderly hemodialysis patients. The results showed that the average score of self-care behavior was the highest in the self-care ability of maintaining arteriovenous Fistula and taking medicine, the self-care ability to participate in social activities and Diet Control scored lowest; a Turkish study found that the self-care ability score of hemodialysis patients was (62.50 \pm 12.20$)$, at a lower level [17]. Self-care ability of MHD patients directly affects their quality of life [18] [19]. Therefore, in order to prolong the survival time of MHD patients and improve their quality of life, it is essential to help them improve their self-care ability. Marcus [20] evaluated the self-management and knowledge of $372 \mathrm{HD}$ patients from 17 dialysis institutions. The results showed that the self-management level of HD patients was low. Curtin [21] scored (66 \pm 4.704) in self-management behavior, and the score of medication compliance was the highest (3.64 \pm 0.805$)$. Ningyunfeng [22] through the investigation and analysis of 95 cases of maintenance HD patients, we found that $45 \%$ of the patients had poor self-management behavior. Fan Jiali et al. [23] investigated 301 HD patients in three top hospitals, the score of self-management was (66.02 \pm 10.59), which was low. Zhang Lin [24], investigated 152 young HD patients in China, the results showed that the average total score of self-management behavior of young HD patients was $(53.91 \pm 10.73)$ [25], which was at a low level, self-management is at a moderate level. Cha, J [26] surveyed 215 people, the score of self-management was $(60.35 \pm 8.18)$, the range of score was (35 - 76), the level of self-management was higher, $39.5 \%$ of them were still working, $44.2 \%$ of them were college graduates, this may also be related to patients receiving more social support. Natashia [27] investigated 145 HD patients with low level of self-management $(74.1 \pm 10.72)$.

\section{Factors Influencing Self-Care Ability of MHD Patients}

\subsection{Demographic Data}

1) Gender Xia Linjuan [28] the results showed that gender has an effect on 
self-management behavior of MHD patients, and the level of self-management behavior of women is higher than that of men. This is consistent with the findings of many scholars abroad: they consider gender to be an important factor in self-care ability; a survey in Iran [2] shows that men have better self-care ability than women, this is consistent with the results of Eylem Bag MsN-RN [29] et al.

2) Age Iran [30] a study has shown that young hemodialysis patients can significantly improve their ability to care for themselves; studies by Heidar Zadeh M [31] have also shown that lower age can improve the quality of life of hemodialysis patients, there was also a significant correlation between quality of life and self-care ability. This is consistent with the findings of Wang Jing and Yang Yanxia [32] [33]; Huo Qianwei [34], who believe that the older the patients are, the more they feel that they are in poor physical condition and the less able they are to care for themselves.

3) The results of the one-way Anova and multiple stepwise regression analysis showed that the self-care ability of hemodialysis patients was influenced by the education level [32], and the self-care ability of hemodialysis patients was influenced by the self-care ability by the education level, there was significant statistical significance $(P<0.05)$. The higher the level of education, the stronger the ability of patients to self-care. Xia Linjuan [28] showed that patients with different educational levels had different total scores of self-management behaviors. The higher the educational level, the higher the total score of self-management behaviors. Patients with higher education level have higher self-care ability, which is consistent with the results of a study in Iran [30]. The reason may be that patients with high education level can consciously obtain diseases and treatment related through various ways. The self-care ability of patients with a higher education level is higher, which is also consistent with the results of a study in Iran [30]. The reason may be that patients with high educational level can acquire knowledge about disease and treatment through various ways, and it is easy to understand and master some self-care knowledge and skills, and actively cooperate with the medical staff to participate in the treatment to have a better self-care capacity.

4) Marital status Iran [30] a study suggests that single individuals are better at self-care than married patients; studies like Wang Jing [18] have found differences in self-care ability among patients with different marital status, married and divorced patients had better self-care ability, while widowed patients had the worst self-care ability, which was inconsistent with the results of Zhang Ming's [35] study: widowed patients had the highest total self-care ability score and divorced patients had the lowest score. Whether marital status has an effect on self-care ability of hemodialysis patients is controversial and needs further study.

\subsection{Disease-Related Factors}

1) Dialysis time Zhu Xiaofeng [36] and other studies showed that the duration of the disease course of hemodialysis patients was negatively correlated with the 
scores of general health and mental health dimensions. The duration of dialysis may affect a patient's ability to self-care. The self-care ability of 195 hemodialysis patients and its influencing factors were analyzed by Wang Jing [32]. The results showed that the self-care ability of hemodialysis patients with different dialysis time was different. The self-concept and self-care knowledge of the 5-year patients were higher than that of the 10 -year patients $(\mathrm{P}<0.05)$. A study in Iran [30] showed that hemodialysis duration had no effect on patients' self-care ability, which may be related to the physical quality of MHD patients in the region or the self-care ability rating scale used.

2) Fatigue has been found to be one of the most common symptoms in MHD patients and an important prognostic factor [37]. Fatigue is a multi-dimensional and complicated subjective feeling, which often causes excessive physical and psychological consumption and causes patients to be extremely tired and accompanied by sleep disorders, physical and physical activity decline, this symptom not only affects the self-care ability of MHD patients, but also reduces their quality of life (Qol) [38]. Zhou Lijuan, et al. [39], mental Fatigue of MHD patients was negatively correlated with the total score of self-management behavior and all dimensions.

\subsection{Psychological Factors}

An Iranian [30] survey on the relationship between anxiety and self-care ability in 140 hemodialysis patients showed that anxiety was an important influence factor on self-care ability after controlling for the influence of dialysis time, the lower anxiety level can enhance the self-care ability of MHD patients and has a positive effect on their health outcomes. There is a significant negative correlation between the quality of life and depression and anxiety in hemodialysis patients; the results show that depression has an effect on self-management behavior, and the more severe the depression, the lower the level of self-management behavior [28].

\subsection{Self-Efficacy}

Wang Zhanglin [40] research shows that improving patients'self-care ability can improve their skills and knowledge level of self-care diseases, enhance their sense of responsibility of self-care, and better manage diseases, [41], there was a positive correlation between self-care ability and self-efficacy in MHD patients, and the self-efficacy level of hemodialysis patients increased when the self-care ability level increased many foreign scholars have found that there is a positive relationship between self-efficacy and self-care ability in hemodialysis patients [42], can improve the patient's self-care ability and quality of life [43]. The correlation between self-efficacy and self-management behavior of 86 hemodialysis patients was investigated [44]. The results of correlation analysis showed that self-efficacy was positively correlated with self-management behavior as a whole and all dimensions, to improve the self-efficacy of patients is helpful to improve 
their self-management level. The correlation between self-management behavior and self-efficacy was investigated in 98 MHD patients who met the inclusion criteria [45], the self-efficacy level of MHD patients was positively correlated with exercise behavior, emotion and symptom management behavior.

\subsection{Other}

Foreign scholars believe that the existence of social support can improve the quality of life of patients and buffer the impact of adversity, improve their happiness; Chan Ym et al. [46] believed that there was a significant positive correlation between self-care behavior and social support in elderly hemodialysis patients; there is a significant positive correlation between self-care behavior and social support in elderly hemodialysis patients [28]. For patients with chronic diseases, social support can strengthen their psychological adjustment, reduce anxiety, depression symptoms and disease needs, and improve their quality of life. The results showed that the higher the level of social support, the higher the level of self-management behavior of MHD patients. The results of Wang Jing's [32] study showed that the average monthly income had no effect on the self-care ability of hemodialysis patients; the results of Yang Yanxia's [33] multiple regression showed that the higher the monthly income, the stronger the self-management ability of hemodialysis patients; the results of Zhang Ming [35] also showed that the scores of self-care ability of patients with different monthly income were different, and the self-care ability of patients with higher monthly income was also higher; Cheng Qiong [47] investigated the influence of economic status of caregivers on self-care ability of 100 hemodialysis patients. The results showed that good economic status of caregivers could promote self-efficacy and improve self-care ability of ESRD patients, reduce negative emotions. Whether the average monthly income of different families has an effect on the self-care ability of patients is controversial and needs further study.

\section{Nursing Intervention Measures to Improve the Mental Health Level of Maintenance Hemodialysis Patients}

1) Health education in most cases, the low mental health level of maintenance hemodialysis patients is related to their lack of relevant knowledge. Through health education, nursing staff can help them to acquire relevant knowledge, change the past and false ideas, to form a correct understanding of the disease, and thus improve their mental health level. The contents of health education include: to introduce the hemodialysis environment, disease-related knowledge, such as the principle of maintenance hemodialysis, treatment effect, the performance and safety of dialysis machine, the protection of internal Fistula, and the dialysis process may appear in the discomfort and matters needing attention and so on, also includes the Diet and takes the medicine instruction and so on. There are three main forms of health education, namely, one-to-one conversation between nurses and patients, expert lectures or patients with good dialysis effect. Methods include oral communication, providing patients with therapeutic pub- 
lications, watching VCD videos, etc. [48] [49]. Studies have shown that maintenance hemodialysis patients with health education can improve their mental health level. He Zhigang et al. [49] through 40 patients with disease knowledge education, the presence of patients, effectively reduce the anxiety of maintenance hemodialysis patients.

2) Family involvement in the family as the basic unit of the social network through the provision of support, information and feedback to help maintenance hemodialysis patients relieve mental stress and maintain their mental integrity 1261 . Therefore, we should attach importance to the positive guidance of family members to the patients with maintenance hemodialysis. Studies have shown that by establishing the necessary contact with the patient's closest or relatively constant accompanying family members, the nursing staff can communicate with the patient at any time about possible or existing psychological problems, and involve the family members in health education activities for the patient, it can significantly improve patients' ability of psychological adaptation to diseases, better treat their psychological symptoms, adapt to dialysis treatment and improve their quality of life [48] [49].

3) Regular physical training system and regular physical training is an important rehabilitation treatment for maintenance hemodialysis patients, aiming at improving their physical function and psychological state. The forms of exercise include whole body aerobic exercise, breathing adjustment exercise, stationary bicycle exercise and so on. Some foreign research has carried on the beneficial exploration to this. A 12-week exercise study in maintenance hemodialysis patients in South Korea confirmed that regular exercise can improve patients' mental state and alleviate depression [50] [51].

4) In the field of psychological intervention, more and more scholars are using supportive therapy, biofeedback technology and cognitive behavioral intervention to improve depression, anxiety, fear and non-compliance in hemodialysis patients, these techniques have also significantly improved the psychological status and quality of life of patients [52]. Cognitive behavioral therapy and interpersonal therapy have been shown to be effective in relieving depressive symptoms. Psychotherapy intervention and physical function training can significantly improve the physiological and psychological state of maintenance hemodialysis patients, and can significantly reduce or alleviate the incidence of depression in maintenance hemodialysis patients, improve patient quality of life [53]. Using individual cognitive behavior therapy to intervene hemodialysis patients [53], we found that psychological stress education and individual counseling can help patients identify the source of stress, it has significant effect on correcting anxiety, depression, psychological and social maladjustment and improving interpersonal relationship. Maintenance hemodialysis patients are suffering from treatment and disease-related factors, and their mental health level is low. At present, the intervention study on the mental health status of maintenance hemodialysis patients is more extensive, but the psychological problems of this population are still not optimistic. The reasons may be related to the lack of 
standardization and systematization of intervention methods and the lack of generalization. Therefore, the feasibility of a number of interventions, effective evaluation of the effectiveness and how to promote the issue of nursing staff still need to actively explore.

\section{Summary at Present}

For chronic disease self-care, the importance of the consensus has been reached at home and abroad, and a large number of studies have been carried out, such as rheumatoid arthritis, diabetes, emotional disorders, chemotherapy, permanent colostomy and other aspects of self-care have been reported, including cross-sectional studies and intervention studies, it is also involved in hemodialysis patients. The study of self-care ability of MHD patients is mostly seen in the study of its influencing factors at home and abroad, and the intervention study is less, and the intervention study both at home and abroad shows that the effective intervention measures play a positive role in improving the self-care ability of MHD patients, but there is no consensus on the content of intervention and evaluation index. MHD patients are a kind of chronic patients who receive special treatment. Self-care plays an important role in their life. Therefore, it is necessary to further explore the intervention program to enhance the self-care ability of MHD patients, so as to improve the self-care of MHD patients and improve their quality of life.

\section{Conflicts of Interest}

The authors declare no conflicts of interest regarding the publication of this paper.

\section{References}

[1] Lu, H., Wu, C., Tang, Y., et al. (2014) Progress in Nursing Research on Self-Management of Maintenance Hemodialysis Patients. New Clinical Medicine in China, 7, 177-180.

[2] Atashpeikar, S., Jalilazar, T. and Heidarzadeh, M. (2012) Self-Care Ability in Hemodialysis Patients. Journal of Caring Sciences, 1, 31-35.

[3] Jiang, A. (2009) Nursing Theory. People's Medical Publishing House, Beijing.

[4] Lu, M., Chen, Y., Zeng, J., et al. (2015) Orem Self-Care Model on the Self-Care Ability and Quality of Life of Stroke Patients. Guangdong Medicine, 36, 2602-2604.

[5] Callaghan, D.M. (2003) Health-Promoting Self-Care Behaviors, Self-Care Self-Efficacy, and Self-Care Agency. Nursing Science Quarterly, 16, 247-254. https://doi.org/10.1177/0894318403016003016

[6] Orem, D.E. (1995) Nursing. Concepts of Practice. Mosby Publishing, St. Louis.

[7] Denyes, M.J., Orem, D.E. and Bekel, G. (2001) Self-Care: A Foundational Science. Nursing Science Quarterly, 14, 48-54. https://doi.org/10.1177/089431840101400113

[8] Kearny, B.Y. and Fleischer, B.J. (1979) Development of an Instrument to Measure Exercise of Self-Care Agency. Research in Nursing and Health, 2, 25-34. https://doi.org/10.1002/nur.4770020105 
[9] Denyes, M. (1980) Development of an Instrument Measure Self-Care Agency in Adolescents. Intervention International, 41, 1716B.

[10] Sousa, V.D., Zauszniewski, J.A., Musil, C.M., et a1. (2005) Relationships among Self-Care Agency, Elfefficacy, Self-Care, and Glycemic Control. Research and Theory for Nursing Practice, 19, 217-230. https://doi.org/10.1891/rtnp.2005.19.3.217

[11] Gantz, S.B. (1990) SELF-CARE: Perspectives from Six Disciplines. Holistic Nursing Practice, 4, 1-12. https://doi.org/10.1097/00004650-199002000-00004

[12] Yang, F., Xu, H.Y., Yang, Y.Y. (2007) Diversified Health Education Improves Self-Care Ability of Dialysis Patients. Journal of Qiqihar Medical College, 28, 2810-2811.

[13] Luo, L., Lei, Y.L., Bao, Y.Q., et al. (2016) Survey on Quality of Life and Self-Care Ability of Maintenance Hemodialysis Patients in Chongqing. Journal of PLA Nursing, 33, 37-39.

[14] Atashpeikar, S., Jalilazar, T. and Heidarzadeh, M. (2012) Self-Care Ability in Hemodialysis Patients. Journal of Caring Sciences, 1, 31-35.

[15] Griva, K., Lam, K.F.Y., Nandakumar, M., et al. (2018) The Effect of Brief Self-Management Interventions for Hemodialysis Patients (HED-SMART) on Trajectories of Depressive and Anxious Symptoms. Journal of Psychosomatic Research, 113, 37-44. https://doi.org/10.1016/j.jpsychores.2018.07.012

[16] Lu, H., Luo, Y.F., Sun, Z.Y. (2013) Nursing Intervention on Self-Care Ability of Elderly Patients with Maintenance Hemodialysis Efficiency Evaluation. Nursing Practice and Research, 10, 24-25.

[17] Mollaoglu, M. (2006) Perceived Social Support, Anxiety, and Self-Care among Patients Receiving Hemodialysis. Dialysis \& Transplantation, 35, 144-155. https://doi.org/10.1002/dat.20002

[18] Mehdi, H., Solmaz, A. and Tahereh, J. (2010) Relationship between Quality of Life and Self-Care Ability in Patients Receiving Hemodialysis. Iranian Journal of Nursing \& AMP: Midwifery Research, 15, 71-76.

[19] Ageborg, M., Allenius, B.-L. and Cederfjäll, C. (2010) Quality of Life, Self-Care Ability, and Sense of Coherence in Hemorrhagic Patients: A Comparative Study. Hemodialysis International, 9, S8-S14. https://doi.org/10.1111/j.1542-4758.2005.01164.x

[20] Wild, M.G., Wallston, K.A., Green, J.A., et al. (2017) The Perceived Medical Condition Self-Management Scale Can Be Applied to Patients with Chronic Kidney Disease. Kidney International, 92, 972-978. https://doi.org/10.1016/j.kint.2017.03.018

[21] Curtin, R.-B., Sitter, D.-C., Schatell, D., et al. (2004) Self-Management, Knowledge, and Functioning and Well-Being of Patients on Hemodialysis. Nephrology Nursing Journal, 31, 378-387, 396.

[22] Ning, Y. (2011) Investigation on Self-Management Ability of Hemodialysis Patients. PLA Journal of Nursing, 28, 19-21.

[23] Fan, J., Kong, Y., Shi, S., et al. (2016) Relationship between Locus of Control and Self-Management Behavior in Hemodialysis Patients. PLA Journal of Nursing, 33, 23-27.

[24] Zhang, L., Zhang, H., Liu, X., et al. (2017) Self-Management Behavior and Its Influencing Factors in Young Hemodialysis Patients. Guangdong Medicine, 38, 459-462.

[25] Ören, B. and Enç, N. (2014) Development and Psychometric Testing of the Self-Care Agency Scale for Patients Undergoing Long-Term Paralysis in Turkey. Journal of 
Renal Care, 40, 266-273. https://doi.org/10.1111/jorc.12098

[26] Cha, J. (2017) Structural Equation Modeling of Self-Management in Patients with hemodialysis. Journal of Korean Academy of Nursing, 47, 14-24.

https://doi.org/10.4040/jkan.2017.47.1.14

[27] Natashia, D., Yen, M., Chen, H.-M., et al. (2019) Self-Management Behaviors in Relation to Psychological Factors and Interdialytic Weight Gain among Patients in Indonesian. Journal of Nursing Scholarship, 51, 417-426. https://doi.org/10.1111/jnu.12464

[28] Xia, L.J. (2014) Study on Self-Management Behavior and Its Influencing Factors in Maintenance Hemodialysis Patients. Tianjin Medical University, Tianjin.

[29] Mollaoglu, M. (2005) Hemodiyaliz Uygulanan Hastalarda Oz BakimGucu ve Depresyon. Nefroloji Hems, Ireligi Dergisi, 12, 55-60.

[30] Yurtsever, S. and Kuytatar, E. (2005) Hemodiyaliz Hastalarmda Yorgun-luk ve Oz Bakim. Nefroloji Hems, Ireligi, 18, 26-32.

[31] Heidar Zadeh, M. (2006) Relationship between Quality of Life and Social Support in Hospitals of Tabriz University of Medical Sciences. Tabriz University of Medical Sciences, Tabriz.

[32] Wang, J. (2016) Self-Care Ability and Its Influencing Factors in Hemodialysis Patients. Shandong University, Jinan.

[33] Yang, Y. (2014) Personality Traits Affecting Self-Management Ability in Hemodialysis Patients. Nanchang University, Nanchang.

[34] Fok, S. and Zou, D. (2005) Evaluation of Self-Care Ability of Hemodialysis Patients in Tianjin. Chinese Journal of Nursing, 40, 251-254.

[35] Zhang, M. (2014) Study on Self-Care Ability and Its Influencing Factors of Tumor Patients during PICC Indwelling. Shandong University, Jinan.

[36] Zhu, X., Zhang, J., Xu, L., et al. (2005) Quality of Life and Related Factors in Maintenance Hemodialysis Patients with End-Stage Renal Disease. Blood Purification in China, 4, 310-313.

[37] Johansen, K.L., Kaysen, G.A., Dalrymple, L.S., et al. (2013) Association of Physical Activity with Survival among Pediatric Patients on Dialysis: The Comprehensive Dialysis Study. Clinical Journal of the American Society of Nephrology, 8, 248-253. https://doi.org/10.2215/CJN.08560812

[38] Picariello, F., Moss-Morris, R., Macdougall, I.C., et al. (2018) Cognitive-Behavioural Therapy (CBT) for Renal Fatigue (BReF): A Feasibility Randomised-Controlled Trial of CBT for the Management of Fatigue in Haemodialysis (HD) Patients. BMJ Open, 8, E020842. https://doi.org/10.1136/bmjopen-2017-020842

[39] Zhou, L., Bian, Y. and Wang, Q. (2017) Relationship between Fatigue and Self-Management Behavior in Maintenance Hemodialysis Patients. Journal of Nursing, 24, $38-41$.

[40] Wang, Z. (2012) Effect of Self-Efficacy Intervention on Self-Care Ability of Maintenance Hemodialysis Patients. Jilin University, Changchun.

[41] Chung, A. (2014) Effect of Self-Efficacy Intervention on Self-Care Ability of Maintenance Hemodialysis Patients. Chinese Medicine Guide, 12, 15-16.

[42] Bağ, E. and Mollaoğlu, M. (2010) The Evaluation of Self-Care and Self-Efficacy in Patients Undergoing Hemodialysis. Journal of Evaluation in Clinical Practice, 16, 605-610.

[43] Cheng, C. (2014) Effect of Self-Efficacy Intervention on Nursing Ability and Quality 
of Life of Hemodialysis Patients. Nursing Practice and Research, No. 12, 44-45.

[44] Shen, X., Zhou, Y., Han, X., et al. (2013) Correlation between Self-Efficacy and Self-Management Behavior in Hemodialysis Patients. Chinese Journal of Modern Nursing, 19, 3326-3329.

[45] Ju, H., Zhang, F., Su, C., et al. (2012) Correlation between Self-Management Behavior and Self-Efficacy in Maintenance Hemodialysis Patients. Chinese Journal of Modern Nursing, 18, 4375-4378.

[46] Chan, Y.M., Zatilah, M.S. and Hii, S.Z. (2012) Determinants of Compliance Behaviors among Patients Going Hemodialysis in Malaysia. PLoS ONE, 7, e41362. https://doi.org/10.1371/journal.pone.0041362

[47] Ching, J. (2016) Influence of Economic Status of Caregivers on Self-Care Ability of Hemodialysis Patients. International Journal of Transplantation and Blood Purification, 14, 39-41.

[48] Zhang, Z. and Liu, J. (2004) Investigation and Analysis on Mental Health Status of Maintenance Hemodialysis Patients in Five General Hospitals in Beijing Area. China Aerospace Medical Journal, 6, 76-79.

[49] He, Z. and Yuan, J. (2004) Coping with Stress in the Nursery. Journal of Nursing, 19, 18-19.

[50] Suh, R.N.M.R., Jung, H.H., Kim, S.B., et al. (2002) Effects of Regular Exercise on Anxiety, Depression, and Quality of Life in Maintenance Hemodialysis Patients. Renal Failure, 24, 337-345. https://doi.org/10.1081/JDI-120005367

[51] Curtin, R.B., Roaj, M.J., Bultman, D.C. and Schatell, D. (2002) Renal Rehabilitation and Improved Patient Outcomes in Texas Dialysis Facilities. American Journal of Kidney Diseases, 40, 331-338. https://doi.org/10.1053/ajkd.2002.34517

[52] Ernst, C.L. and Goldberg, J.F. (2003) Antidepressant Properties of Anticonvulsant Drugs for Bipolar Disorder. Journal of Clinical Psychopharmacology, 23, 182-192. https://doi.org/10.1097/00004714-200304000-00011

[53] Levendoglu F., Altintepe, L., Okudan, N., et al. (2004) A Twelve Week Exercise Program Provides the Psychological Requirements for Patients. Journal of Nephrology, 17, 826-832. 